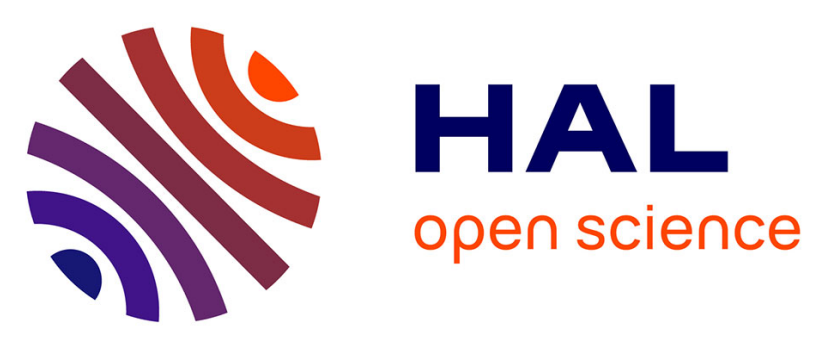

\title{
Dynamic instabilities of rod-like eutectic growth patterns: A real-time study
}

Mikael Perrut, Sabine Bottin-Rousseau, Gabriel Faivre, Silvère Akamatsu

\section{To cite this version:}

Mikael Perrut, Sabine Bottin-Rousseau, Gabriel Faivre, Silvère Akamatsu. Dynamic instabilities of rod-like eutectic growth patterns: A real-time study. Acta Materialia, 2013, 61, pp.6802 - 6808 . 10.1016/j.actamat.2013.07.054 . hal-01448377

\section{HAL Id: hal-01448377 \\ https://hal.science/hal-01448377}

Submitted on 23 Feb 2017

HAL is a multi-disciplinary open access archive for the deposit and dissemination of scientific research documents, whether they are published or not. The documents may come from teaching and research institutions in France or abroad, or from public or private research centers.
L'archive ouverte pluridisciplinaire HAL, est destinée au dépôt et à la diffusion de documents scientifiques de niveau recherche, publiés ou non, émanant des établissements d'enseignement et de recherche français ou étrangers, des laboratoires publics ou privés. 


\title{
Dynamical instabilities of rodlike eutectic growth patterns: A real-time study
}

\author{
Mikaël Perrut ${ }^{\mathrm{a}}$, Sabine Bottin-Rousseau, Gabriel Faivre, Silvère Akamatsu* \\ Institut des Nanosciences de Paris, UPMC, CNRS, 4 place Jussieu, 75252 Paris Cedex 05, France \\ a Present Address: Office National d'Etudes et Recherches Aérospatiales, 29 avenue de la \\ Division Leclerc, 92322 Chatillon, France
}

\begin{abstract}
We present a real-time experimental study of the rodlike growth patterns formed during directional solidification in a non-faceted transparent eutectic alloy, succinonitrile(d)camphor. Slightly convex isotherms were used to slowly increase the pattern spacing $\lambda$ from an appropriate starting value to the threshold spacing for the rod elimination or rod splitting instabilities allowing a quantitative determination of these thresholds as a function of the solidification rate $V$. We show that the threshold spacing for rod splitting obeys the general $\lambda \sim V^{-1 / 2}$ scaling law of eutectic growth whereas the threshold spacing for rod elimination deviates from this law at low $V$, exhibiting the same overstability effect as that previously reported for lamellar eutectic patterns. We demonstrate that topological defects (walls between hexagon domains) play an important role in rod splitting processes. We also describe a spatially incoherent mode of oscillation that we observed in disordered rodlike patterns.
\end{abstract}

Key words: Eutectic solidification, directional solidification, solidification microstructures

\section{Introduction}

Nonfaceted eutectic growth is one of the oldest known experimental example of $2 \mathrm{D}$ pattern-forming out-of-equilibrium system $[1,2,3]$. It shares the same

\footnotetext{
*Corresponding author

Email address: akamatsu@insp.jussieu.fr()
} 
translational and rotational symmetry as, for instance, Rayleigh-Benard convection experiments and, like the latter, is multistable [4]. At given control parameters, it can settle in space-periodic stationary patterns either lamellar (periodic in one direction) or rodlike (consisting of 2D periodic arrays). The spacing and orientation of these patterns are not sharply selected but are boundary condition- and history-dependent. Various "defective" patterns, ranging from space-periodic patterns traversed by a few topological defects to fully disordered patterns, can also be more or less stationary. The selection processes between these different types of growth patterns as a function of boundary conditions and solidification history have not yet been fully elucidated. The investigation of such processes mostly relies on real-time observations of well-controlled systems over sufficiently long period of times. Because eutectic patterns are by their nature immersed in a solidifying melt, and, moreover, usually have spacing values $(\lambda)$ in the micrometer range, their in-situ study has long been limited to quasi 1D systems (leading, incidentally, to an almost complete elucidation of the dynamics of such systems $[5,6])$. It is only a few years ago that an experimental method of observing insitu 2D eutectic patterns in transparent alloys was proposed [7]. This method was applied to lamellar eutectic patterns, revealing that the stability of such patterns is limited by a zigzag bifurcation followed by lamella disruption and branching on the large- $\lambda$ side [8], in agreement with the results of phase-field numerical studies [9]. It was also found that a necessary condition for lamellar patterns to form was the use of a solidification setup with a slight misalignment of the direction of growth and the axis of the thermal gradient. Without this additional ingredient, called "thermal bias", the eutectic growth patterns remained indefinitely labyrinthic [10].

The same method of in-situ observation is currently being used to study the dynamics of rodlike eutectic patterns. The experiments are performed in the transparent alloy succinonitrile-(d)camphor (SCN-DC), which is known to be a nonfaceted rodlike eutectic $[11,12]$. The question of the long-term growth regime of this system was addressed in a previous report, which pointed out the crucial role played by "imperfections" of the experimental setup in the selection of this regime [13]. More specifically, it was shown that a slight forward curvature of the isotherms drove the rodlike pattern towards an operating point close to its large- $\lambda$ stability limit, which corresponds to a rod splitting instability. During this study, we used the same mild external forcing to study quantitatively the threshold spacings for both the rod splitting and the rod elimination instabilities, the latter corresponding to the small- $\lambda$ stability limit of the growth patterns. Although curved isotherms are a common feature in bulk directional solidification, it is only 
relatively recently that their effects on growth patterns have begun to be studied in detail $[14,15,16]$. Before presenting our findings about the instability threshold spacings, we shall therefore sum up our previous conclusions about the effects of convex isotherms on the dynamics of rodlike eutectic patterns.

\section{Experimental Methods}

The eutectic point of the SCN-DC alloy is at $T_{E}=38.3^{\circ} \mathrm{C}$ and $C_{E}=13.9$ mol\%DC [11]. The eutectic plateau connects an almost pure-SCN body centered cubic phase and an almost pure-DC hexagonal phase. Thereafter, these phases are called SCN and DC, respectively. The alloys we used were within less than $\pm 0.1 \mathrm{~mol} \%$ of $C_{E}$. A solid at this concentration has a DC volume fraction of about 0.23 and grows from the melt by forming a rodlike eutectic pattern, with rods of the DC phase embedded in a SCN matrix [12]. Directional-solidification experiments were conducted as follows. The alloy is put in cartridges made of two 300 mm-thick glass plates separated by plastic spacers delimiting an empty space of cross section $6 \times 0.4 \mathrm{~mm}^{2}$. A device acting as grain selector is placed near one end of the cartridges allowing one to grow single, or at least large, eutectic grains. A filled cartridge is placed between a cold oven and a hot oven adjusted to give a thermal gradient of $8 \pm 1 \mathrm{Kmm}^{-1}$ in the region of interest. Solidification is carried out by pulling the sample towards the cold end of the gradient at a constant rate $V$. Let $\mathbf{z}$ be the direction opposite to that of the pulling, $\mathbf{y}$ the direction perpendicular to the sample plane and $\mathbf{x}$ the direction perpendicular to $\mathbf{y}$ and $\mathbf{z}$. In theory, $\mathbf{z}$ coincides with the axis of the thermal gradient and the isotherms are planes perpendicular to $\mathbf{z}$. In practice, the isotherms are generally slightly curved and tilted with respect to $\mathbf{z}$.

During the pulling, the average position of the solid-liquid interface remains locked onto an isotherm close to $T_{E}$ and is observed with an optical microscope. We use a dark-field method based on the fact that the three (SCN, DC, liquid) phases of interest have different refractive indices. This method can be briefly described as follows (For details, see [7]). The solid-liquid interface is observed from the outside of the crucible through the liquid under oblique view. The angle between the $\mathbf{y}$ axis and the direction of observation is of about $40^{\circ}$. The solid is illuminated obliquely and slightly from below. The light rays that travel along a DC rod are refracted by the curved interface ("DC cap") between this rod and the liquid to form a caustic at a short distance above the interface. The images collected essentially consist of arrays of bright spots, each of which represents the caustic associated to a DC cap (Fig. 1). The as-received images are processed 
for subtracting background and stretched in the $\mathbf{y}$ direction in order to correct for the contraction due to the inclination of the direction of observation. The resolution limit of this method is of about $3 \mu \mathrm{m}$. It is well known that the order of magnitude of the spacing of a eutectic pattern is given by the Jackson-Hunt scaling law $\lambda \sim \lambda_{J H}=\sqrt{K_{J H}} V^{-1 / 2}$, where $K_{J H}$ is a material constant [3]. In near-eutectic SCN-DC, $K_{J H}=10.2 \pm 1.5 \mu \mathrm{m}^{3} \mathrm{~s}^{-1}$ [12]. During this study, the $V$-range used was of 0.018 to $0.25 \mu \mathrm{ms}^{-1}$, corresponding roughly to a $\lambda$-range of 5 to $30 \mu \mathrm{m}$.

Following common practice, we shall present the measurements of threshold spacings in terms of the dimensionless ("reduced") spacing $\Lambda=\lambda / \lambda_{J H}$. When illustrating local instability processes, we shall make use of Voronoi diagrams with a gray scale ranging from white to black as the number of sides of the Voronoi cells ranges from 4 to 8 . With this representation, hexagon domains appear as medium-grey areas, while domain walls mostly consist of strings of penta (lightgrey )-hepta (dark-grey) pairs of cells (Fig. 2). We define the local spacing $\lambda(t)$ of a particular DC cap in a rodlike pattern as the average of the distances between this DC cap and its nearest neighbors at time $t$, as determined by the Voronoi construction. A number of other local parameters, such as the distances and angular orientations of the different nearest neighbors, are of course needed for a complete geometrical description of a rod splitting or rod elimination event in an irregular environment. Empirically, however, we found that a single parameter, namely, $\lambda(t)$, was sufficient for a study of the dynamics of these events under the conditions of our experiments [17]. We therefore limit ourselves to this parameter thereafter. We define the mean spacing $\bar{\lambda}(t)$ as the average of the local spacings of all the DC rods observed at time $t$. The experimental uncertainty on each individual measurement of $\lambda(t)$ was of about $\pm 1.5 \mu \mathrm{m}$. For an essentially hexagonal (more exactly, triangular) arrangement of the rods, $\bar{\lambda} \approx\left[A^{-1} N(t) \sin \pi / 3\right]^{-1 / 2}$, where $A$ is the area of the observation window and $N(t)$ is the number of rods in this window. Since $N(t)>300$ in our experiments, the uncertainty on $\bar{\lambda}$ was negligible.

\section{Directional solidification with convex isotherms}

In our experiments, the $T_{E}$-isotherms were flat in the $\mathbf{x}$ direction, but slightly convex (bulging into the liquid) in the $\mathbf{y}$ direction due to the differences in thermal conductivities of the various materials of which the samples were composed $[13,17]$. The corresponding radius of curvature $R$ was measured to range between 2.5 and $5 \mathrm{~mm}$ in the different samples used. Due to the fact that DC rods (i.e. the trajectories of the DC caps) remain approximately perpendicular to the envelope of the growth front during solidification, the growth patterns, whatever 
their intrinsic dynamics, were continually stretched in the $\mathbf{y}$ direction at a rate $V / R$. Consequently, when the pattern was quiescent (i.e. as long as no rod creation or rod elimination events took place, except of course at the sample walls), the mean spacing increased over time according to

$$
\bar{\lambda}(t)=\bar{\lambda}_{o} e^{t / \tau}
$$

where $\tau=2 R / V$ and $\bar{\lambda}_{o}$ is a constant. As $R$ was much larger than all the characteristic distances of the growth patterns, $\tau$ was long compared to the characteristic times of the processes of interest.

Let $\lambda_{s}(V)$ and $\lambda_{c}(V)$ be the threshold spacings for the rod splitting and rod elimination instabilities, respectively. These quantities are not uniquely defined when the rodlike pattern is disordered (see below) but we may neglect this fact for the moment. Let us consider a long-duration solidification run at constant $V$ starting from $\bar{\lambda}_{o}<\lambda_{c}$. Such a run can be divided into four stages (Fig. 3a). During Stage I, rod elimination is active and $\bar{\lambda}$ increases rapidly. Observations showed that, during this stage, the growth pattern is very disordered and the width of the $\lambda$-distribution is large. Rod elimination stops completely when the lower tail of this distribution is above $\lambda_{c}$.

During Stage II, the pattern is quiescent and $\bar{\lambda}$ rises exponentially, as explained above. Simultaneously, the degree of order of the pattern increases. After a sufficiently long solidification time, the growth pattern becomes organized in hexagon domains close to either of two particular orientations, namely, the H-orientation, which has a nearest-neighbor direction parallel to the sample walls, and the Vorientation, which has a nearest-neighbor direction perpendicular to the sample walls (Fig. 2). The formation process of such structures will not be considered here. We simply note that they are certainly linked to wall and thermal-bias effects, as are also the structures perpendicular to samples walls in lamellar eutectics [10]. Near the end of Stage II, the hexagon domains were about 10 $\lambda$ in size and were separated from each other by almost ideally thin walls. The hexagons were then substantially elongated (by about 20\%) in the y direction. This is the highest degree of order that was observed during this study.

Stage III corresponds to the gradual increase of the rod splitting frequency from zero to the value $\left(R^{-1} V\right.$ per rod) at which it balances out the stretching effect due to convex isotherms. Finally, Stage IV corresponds to a statistically steady, indefinitely long, growth regime, in which the rod splitting frequency and the mean spacing remain constant, or, more exactly, fluctuate about constant values. In fact, during Stage IV, the whole histogram of local spacings averaged on relatively short time scales and, in particular, its mean value $<\bar{\Lambda}>$ are constant and 
history-independent (Fig. 4). Experiments performed at different solidification rates yielded $\langle\bar{\Lambda}\rangle=1.03 \pm 0.04$, indicating that $\left\langle\bar{\Lambda}>\right.$ obeys the $\lambda \sim V^{-1 / 2}$ scaling law. Finally, we note that, during Stages III and IV, the growth pattern retains a structure in $\mathrm{V}$ - and $\mathrm{H}$ - domains in spite of the recurring rod-splitting events. However, the size of the domains is smaller and the domain walls are more diffuse than in Stage II. Examples are given below.

\section{The rod splitting instability}

The solidification rate programs we used during this study are sketched in Fig. $3 b$. After a long transient in a quiescent state at constant $V$, we observed rod splitting events to occur either inside hexagon domains or in domain walls. Voronoi analyses of intra-domain rod splitting processes are shown in Figure 5. The most noteworthy facts are as follows. We noted no important difference in rod splitting processes between $\mathrm{V}$ - and H-domains. In both cases, the rod splitting events occurred in quick succession at neighboring sites, which suggests the existence of a cooperative mechanism (cascade) with a propagation rate on the same order of magnitude as $V$. The value of $\Lambda$ when the splitting occurred was of $1.15 \pm 0.02$ and the deformation of the hexagons in the $\mathbf{y}$ direction was of about $25 \%$. The direction of splitting (i.e. the direction of the vector between the two daughter branches resulting from the splitting of a rod) was that of the most elongated nearest-neighbor direction. After the passage of a rod splitting cascade, the hexagonal ordering of the domains was restored at a smaller spacing $(\approx 1.11)$. To sum up, the intra-domain rod splitting process is the closest approximation we could observe to an instability in a perfect hexagonal rodlike pattern. The observations suggest that a hexagonal rodlike pattern, when subjected to unidirectional deformation, becomes unstable against rod splitting for a deformation slightly less than $25 \%$, or, in terms of local spacing, at $\Lambda_{s}^{h d}$ (hd: hexagon domain) slightly smaller than 1.15 .

Rod splitting processes located in domain walls are shown in Figure 6. The most characteristic feature of such processes is that they only affected rods with particularly large $\Lambda$-values. These rods were always located inside domain walls and were mostly, but not exclusively, represented by heptacoordinated cells in the Voronoi constructions. In the examples under consideration, the average spacing inside the hexagon domains was of about 1.05, and was thus clearly smaller than $\Lambda_{s}^{\text {hd }}$, whereas the local spacing of the rods that were about to split was substantially larger than 1.1 (see below). It should also be noted that the sequencing of splitting events did not suggest that a cooperative mechanism was at play. In other words, 
the different rod splitting events seemed to occur independently from each other, supporting the view of a strictly localized mode of splitting. This mode of splitting was largely predominant in Stage IV. Broadly speaking, its localized character explains the persistence of a multidomain structure with a small grain-size and diffuse walls during this stage.

Figure $7 \mathrm{a}$ shows the time evolution of the local spacing of a rod during a splitting process. The measured data clearly deviated from a $\Lambda(t)=\Lambda_{o} e^{t / \tau}$ law after a time point at which $\Lambda \approx 1.14$. Thus $\Lambda_{s}$ was smaller or equal to that value. No theory of morphological instabilities in disordered hexagonal patterns being available, we fitted various elementary models of instability processes to the data points in order to obtain a lower bound for $\Lambda_{s}$. In brief, we concluded that $\Lambda_{s}=1.2 \pm 0.1$.

During the transients leading up to the rod splitting events, we observed a gradual ovalization of the optical images of the rods, which was undoubtedly due to an elongation of the DC rods that were about to split (Figs. 6b and 7b). This rod elongation process coincided in time with the transitory deviation of the local spacing from a $e^{t / \tau}$ law, and stopped immediately after the splitting had occurred and is therefore clearly part of the instability process itself. It has no obvious link with the stationary rod elongations that exist in stable non-equilateral hexagonal patterns [18].

\section{The rod elimination instability}

The initial condition $\bar{\lambda}_{o}<\lambda_{c}$ was implemented by applying a downward $V$ jump (See Section 3). Rod elimination started at a fast rate almost immediately after the $V$-jump and led to fully disordered growth patterns through a process that could not be resolved with our method of observation. Observable isolated rod elimination events continued to occur in this disordered state for some time (Fig. 8). These events were not accompanied by any detectable rearrangement of the pattern beyond the nearest neighbors of the eliminated rod. In particular, we did not observe any analogue of the phase-diffusion dynamics that is, in theory, the precursor to rod elimination in regular hexagon patterns [19]. Figure 9 shows a $\bar{\Lambda}(t)$-curve measured after a downward $V$ - jump together with the best-fit exponential curve to the data points of Stage II. The spacing value $\bar{\Lambda}_{c}(0.78 \pm 0.03$, in this example) at the time when the data points stop to deviate significantly from the exponential curve gives us a reasonable (over)estimate of the average local value of the rod elimination threshold spacing in disordered states. 
Figure 10 displays $\bar{\Lambda}_{c}$-values measured at different values of $V$. It is striking that $\bar{\Lambda}_{c}$, contrary to $\Lambda_{s}$, does not follow the $\lambda \sim V^{-1 / 2}$ scaling law, but decreases rapidly as $V$ decreases. A similar effect was recently demonstrated, both experimentally and numerically, for lamellar eutectic patterns [20, 21] and was called "overstability" in reference to the fact that $\Lambda_{c}=1$ within the usual normal-growth approximation [22]. A complete theory of the overstability effect is still lacking, but it was argued that $\Lambda_{c}$ should obey the following semi-empirical formula:

$$
1-\frac{1}{\Lambda_{c}^{2}}+B \frac{G \Lambda_{c}}{K_{r} V}=0
$$

where $G$ is the thermal gradient, $K_{r}$ is a material constant and $B$ is an adjustable constant on the order of 0.1 . In near-eutectic SCN-DC, $K_{r}=0.028 \mathrm{Ks} \mu \mathrm{m}^{-2}$ [12]. Least-squares fitting of Eq. (2) onto experimental and numerical data for various lamellar eutectic alloys yielded values of $B$ ranging from 0.067 to 0.15 $[21,12,23]$. As shown in Fig. 10, Eq. (2) with $B=0.075 \pm 0.03$ (including the uncertainties on the values of $G$ and $K_{r}$ ) fits the experimental data for the rod elimination threshold reasonably well. This value of $B$ falls in the range of values found for lamellar patterns, indicating that the overstability effect has the same physical origin in disordered rodlike patterns as in regular lamellar eutectic patterns. In other words, the overstability effect seems to be essentially insensitive to the geometrical particulars of the patterns. It is therefore reasonable to argue that the results shown in Fig. 10 should apply, at least semi-quantitatively, to regular rodlike patterns as well.

\section{Oscillations of disordered rodlike patterns}

Two kinds of spatially coherent modes of oscillation have recently been reported in hexagonal patterns. One was a "sublattice" (or period-tripling) oscillation, found in hexagonal cellular solidification patterns of dilute alloys [24, 25]; the other was a "row" oscillation, occurring in a hexagonal liquid column array [26]. A variety of other coherent modes of oscillation are in theory possible in hexagonal patterns, but have not yet been observed experimental or numerically [27]. We looked for, but did not find any spatially coherent mode of oscillation in rodlike patterns during this study. There are several possible reasons for this, among which an insufficient size of the hexagon domains in our system. Surprisingly, however, we observed a spatially incoherent mode of oscillation which lasted for the duration of Stage IV in two independent experiments performed at $V=0.018 \mu \mathrm{ms}^{-1}$, which was the lowest $V$-value we used. The two experiments 
gave similar results. We limit ourselves here to a brief characterization of this mode of oscillation.

A snapshot and a local space-time diagram of a disordered oscillatory rodlike pattern are shown in Fig. 11. It can be seen that the oscillation manifested itself mostly through an essentially periodic, although somewhat irregular, variation in brightness of the image of each DC rod. A closer analysis showed that this was accompanied by a small-amplitude periodic displacement of the images, indicating that the variation in brightness was in fact mostly due to a variation in the angle with $\mathbf{z}$ of the rods. A rod-by-rod Fourier analysis of the oscillation revealed that the time period $T$ varied slightly from rod to rod. It ranged from 19 to $23.5 \mathrm{~min}$, and was thus close to the value of $\langle\bar{\lambda}\rangle / V(\approx 22 \mathrm{~min})$. This is most probably meaningful given that the time period of the $1 \lambda \mathrm{O}$ oscillations of lamellar eutectic patterns obeys a $T \sim \lambda / V$ scaling law (See Fig. 30 of Ref. [6]). There was no obvious spatial correlation between the differences in period, phase or amplitude of the oscillations of the different rods. There has been no previous report of phenomena related to these spatially incoherent oscillations, as far as we know, except perhaps for the transient oscillation localized on penta-hepta pairs that was noted by Pirat and Gil in imperfect hexagonal liquid column array [26].

\section{Conclusion}

The main conclusions of this study are summed up in Fig. 12. First, the threshold spacing for rod splitting obeys a $\lambda \sim V^{-1 / 2}$ scaling law and is clearly above the Jackson-Hunt scaling length (which corresponds to $\Lambda=1$ ). Second, the threshold spacing for rod elimination exhibits a large deviation from the $\lambda \sim V^{-1 / 2}$ scaling law at low $V$ (overstability) in conformity with Eq. (2). The best-fit value of the empirical constant appearing in this equation $(B=0.075)$ is comparable to those previously found in lamellar eutectic patterns. Third, the steady-state value of the mean spacing during the last stage of directional solidification with convex isotherms is significantly smaller than $\lambda_{s}$. This is consistent with the fact that, in this stage, rod splitting is localized in domain walls and, more precisely, only affects those rods, which have a much larger local spacing than average. Fourth, fully disordered rodlike patterns exhibit, under conditions which remain to be clarified, a spatially incoherent mode of oscillation, whose time period $T$ probably obeys a $T \sim \lambda / V$ scaling law.

It should be kept in mind that these results were established in multidomain rodlike eutectic patterns, for which no theory of morphological instabilities is available yet. As mentioned above, there are reasons to believe that the thresh- 
old spacings for rod splitting and rod elimination in perfect hexagonal rodlike patterns are not very different from those presented here, but this is not certain. It now seems possible, and would indeed be very interesting, to study this question, and, more generally, explore the dynamics of multidomain rodlike patterns, by phase-field simulations [18]. Future experimental studies should undertake to grow single-domain rodlike eutectic patterns using thicker samples and appropriate solidification rate programs [13, 28, 29].

\section{Acknowledgments}

We warmly thank V. T. Witusiewicz, L. Sturz, and S. Rex from ACCESS (Aachen, Germany) for kindly providing purified succinonitrile-(d)camphor alloy and for useful advices. We are glad to acknowledge many fruitful discussions with M. Şerefoğlu and M. Plapp. This work was supported by the Centre National d' Etudes Spatiales, France.

\section{References}

[1] Vogel R. Z. Anorg. Chem 1912;76:550.

[2] Chadwick GA. Prog. in Mater. Sci. 1963;12:97.

[3] Jackson KA, Hunt JD. Trans Metall Soc AIME 1966;236:1129.

[4] Manneville P. Dissipatives structures and weak turbulence. Boston: Academic Press, 1990.

[5] Karma A, Sarkissian A. Met. Trans. A 1996 ; 27: 635.

[6] Ginibre M, Akamatsu S, and Faivre G. Phys. Rev. E 1997;56:780.

[7] Bottin-Rousseau S, Perrut M, Picard C, Akamatsu S, and Faivre G. J. Cryst. Growth 2007;306:465.

[8] Akamatsu S, Bottin-Rousseau S, Faivre G. Phys Rev Lett 2004;93:175701.

[9] Parisi A., Plapp M. Acta Mater. 2008;56:1348.

[10] Perrut M, A. Parisi, Akamatsu S, Bottin-Rousseau S, Faivre G, and Plapp M. Acta Mater. 2010;58:1761.

[11] Witusiewicz V, Sturz L, Hecht U, and Rex S. Acta Mater. 2004;52:5071. 
[12] Akamatsu S, Bottin-Rousseau S, Perrut M, Faivre G, Sturz L, and Witusiewicz V. J. Cryst. Growth 2007;299: 418.

[13] Perrut M, Akamatsu S, Bottin-Rousseau S, and Faivre G. Phys. Rev. E 2009,79:032602.

[14] Bottin-Rousseau S and Pocheau A. Phys. Rev. Lett. 2001;87:076101.

[15] Pocheau A and Bottin-Rousseau S. Chaos 2004;14:882.

[16] Weiss C, Bergeon N, Mangelinck-Noël N and Billia B. Mat. Sci. Engrs A 2005;414-415:296.

[17] Perrut M. Doctoral Thesis, Université Pierre-et-Marie-Curie, 2007.

[18] Şerefoğlu S, Napolitano RE, and Plapp M. Phys. Rev. E 2011;84: 011614.

[19] Caroli B, Caroli C, Roulet B. J. Cryst. Growth 1984;68:677.

[20] Akamatsu S, Plapp M, Faivre G and Karma A. Phys. Rev. E 2002;66:030501.

[21] Akamatsu S, Plapp M, Faivre G, Karma A. Metall Mat Trans A 2004;35:1815.

[22] Langer J.S. Phys Rev Lett 1980; 44:1023.

[23] Akamatsu S, Bottin-Rousseau S, Faivre G. Acta Mater 2011;59:7586.

[24] Plapp M, Dejmek M. Europhys. Lett. 2004;65:276.

[25] Bergeon N, Tourret D, Chen L, Debierre JM, Guérin R, Ramirez A, Billia B, Karma A and Trivedi R. Phys. Rev. Lett. 2013;110:226102.

[26] Pirat C, Mathis C, Massa P, and Gil L. Phys. Rev. Lett. 2004;92:104501.

[27] Pirat C, and Gil L. Physica D 2003;179:92.

[28] Ratke L and Alkemper J. Acta Mater. 2000;48:1939.

[29] Ludwig A, Mogeritsch J, Kolbe M, Zimmermann G, Sturz L, Bergeon N, Billia G, Faivre G, Akamatsu S, Bottin-Rousseau S, and Voss D. JOM 2012;69:1097. 


\section{List of figure captions}

Figure 1: Top view of a rodlike eutectic pattern during directional solidification of a near-eutectic SCN-DC alloy. $V=0.035 \mu \mathrm{ms}^{-1}$. The bright spots are the optical images of the DC rods. The upper and lower edges of the field of view correspond to the inner faces of the crucible. Vertical dimension of the snapshot: $400 \mu \mathrm{m}$. Insert: Schematic 3D drawing.

Figure 2: Schematic Voronoi representation of a multidomain rodlike eutectic pattern. $\mathrm{H}$ and $\mathrm{V}$ refer to the orientations of the hexagon domains. (See the text).

Figure 3: (a) Definition of the successive stages of a long-duration solidification run at constant $V . \bar{\lambda}$ : mean spacing. $\lambda_{c}$ : rod elimination threshold. $\lambda_{s}$ : rod splitting threshold. Dash line: $\bar{\lambda}(t)=\bar{\lambda}_{0} e^{t / \tau}$. (b) Solidification rate programs for the study the rod splitting (1) and rod elimination (2) instabilities. Hatched area: range without rod splitting or elimination. 
Figure 4: Histogram of local spacings averaged over a short period of time $(\approx 0.02 \tau)$ during the last stage of a long-duration solidification run [13, 17]. $\langle\bar{\Lambda}\rangle$ : mean value. $\Lambda_{s}$ : rod splitting threshold (this study).

Figure 5: Intra-domain rod splitting processes. From left to right: Voronoi diagrams of two micrographs taken immediately before and after the process, respectively. The rod splitting events have been labeled alphabetically in chronological order. (a) V-domain. $V=0.11 \mu \mathrm{ms}^{-1}$. Time interval between snapshots: 2 min. Horizontal dimension of a snapshot: $55 \mu \mathrm{m}$. (b) H-domain. $V$ $=0.053 \mu \mathrm{ms}^{-1}$. Time interval between snapshots: $98 \mathrm{~min}$. Horizontal dimension of a snapshot: $140 \mu \mathrm{m}$.

Figure 6: Rod splitting process located in domain walls. $V=0.035 \mu \mathrm{ms}^{-1}$. (a) Same representation as in Fig. 5. Time interval between snapshots: 66min. Horizontal dimension of a snapshot: $150 \mu \mathrm{m}$. (b) Successive snapshots of a single rod splitting process. From left to right: $t=0,14,21$, 28min. Horizontal dimension of a snapshot: $65 \mu \mathrm{m}$.

Figure 7: (a) Time evolution of the local spacing of a DC rod during a rod splitting process. $V$ $=0.035 \mu \mathrm{ms}^{-1}$. Symbols: measured values. Continuous line: $\Lambda=\Lambda_{o} e^{t / \tau}\left(\Lambda_{o}=1.096 ; \tau=\right.$ $\left.2.4 \times 10^{3} \mathrm{~min}\right) . \Lambda_{s}$ : Largest possible value for the rod splitting threshold. (b) Time evolution of the ovalization (aspect ratio minus 1 ) of the optical image of the DC rod. The nonzero $(\approx 0.5)$ baseline is due a residual misalignment of the optical system.

Figure 8: Successive snapshots of an isolated rod elimination event following a $V$-jump from 0.042 to $0.028 \mu \mathrm{ms}^{-1}$. From left to right: $t=0,50,100 \mathrm{~min}$. Horizontal dimension of a snapshot: $50 \mu \mathrm{m}$.

Figure 9: Mean spacing as a function of time after a $V$-jump from 0.071 to $0.042 \mu \mathrm{ms}^{-1}$ applied at $t=0$. Symbols: measured values. Continuous line: $\Lambda=\Lambda_{o} e^{t / \tau}\left(\Lambda_{o}=0.768, \tau=3.3 \times 10^{3} \mathrm{~min}\right)$. $\bar{\Lambda}_{c}$ : rod elimination threshold. 
Figure 10: Rod elimination threshold spacing as a function of solidification rate. Symbols: measured values. Continuous line: Eq. (2) with $B=0.075$.

Figure 11: (a) Snapshot of a disordered oscillating rodlike pattern. $V=0.018 \mu \mathrm{ms}^{-1}$. Horizontal dimension: $440 \mu$. (b) Time evolution of the intensity distribution along a $180 \mu \mathrm{m}-$ long, 1pixelthick area. The rods that are apparently created (eliminated) are in fact entering (leaving) the selected area.

Figure 12: Morphology diagram of multidomain rodlike eutectic patterns in near-eutectic SCNDC alloys. Hatched area: range without rod creation or elimination. Square symbols: oscillatory patterns. $<\bar{\Lambda}>$ : mean spacing at long times in the presence of convex isotherms. 


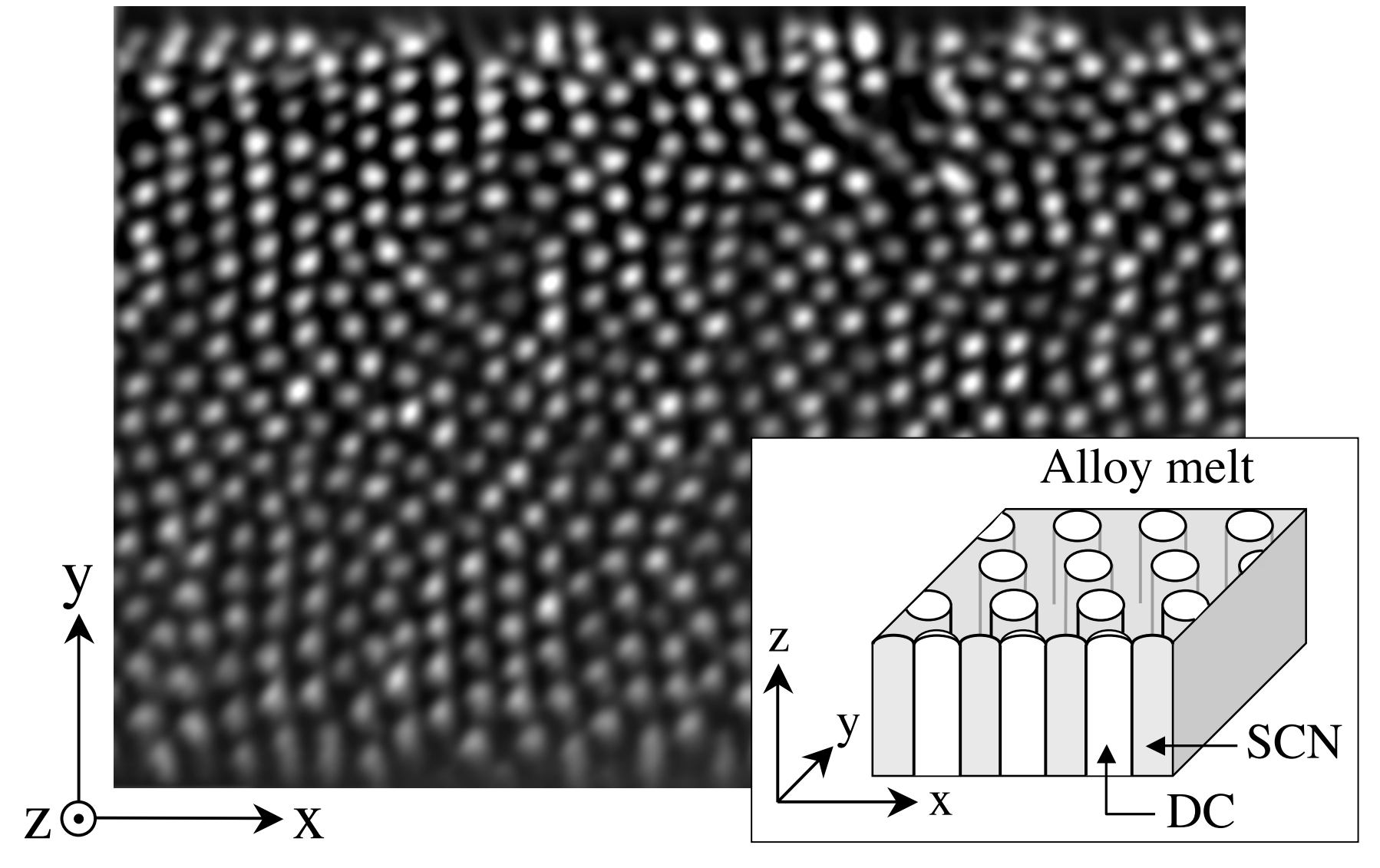




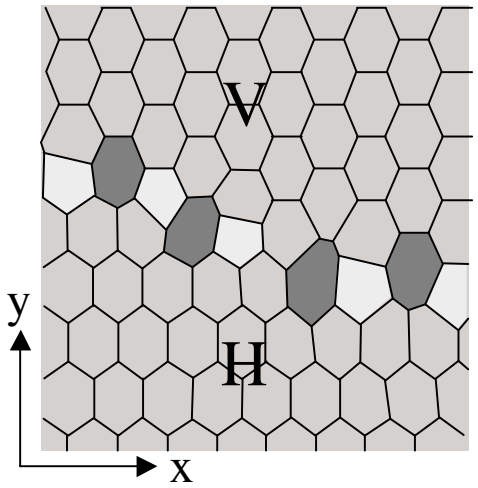




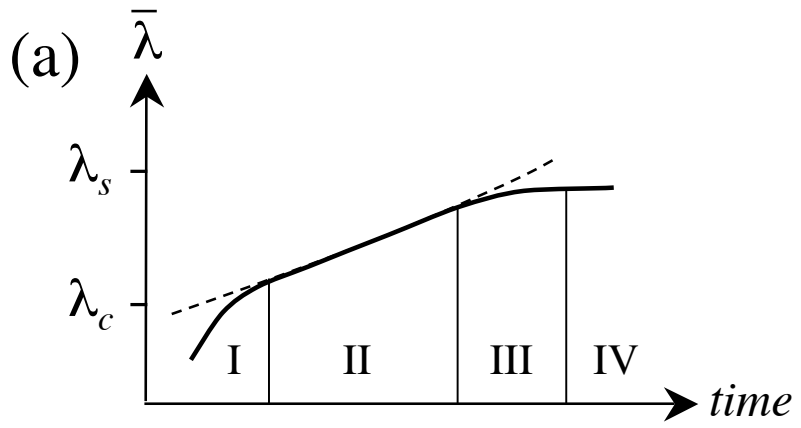

(b) $\mathrm{V}$

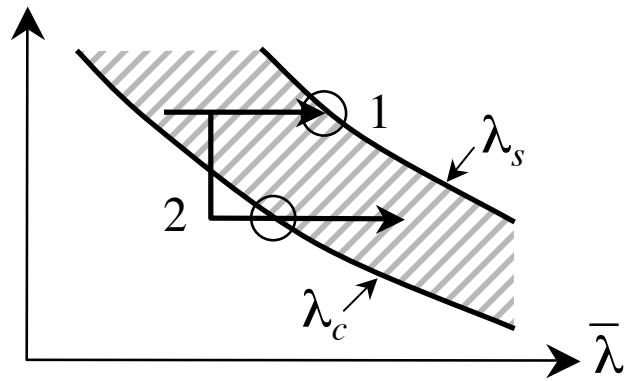




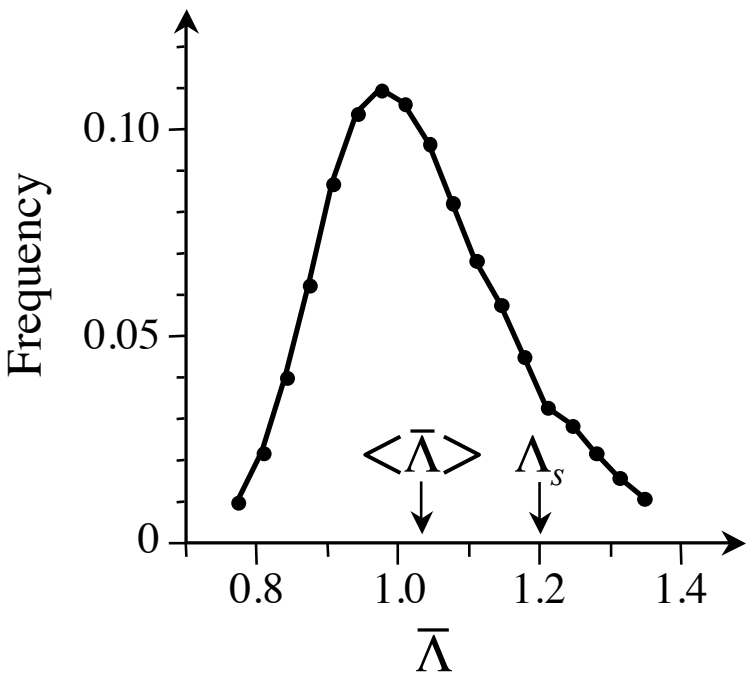



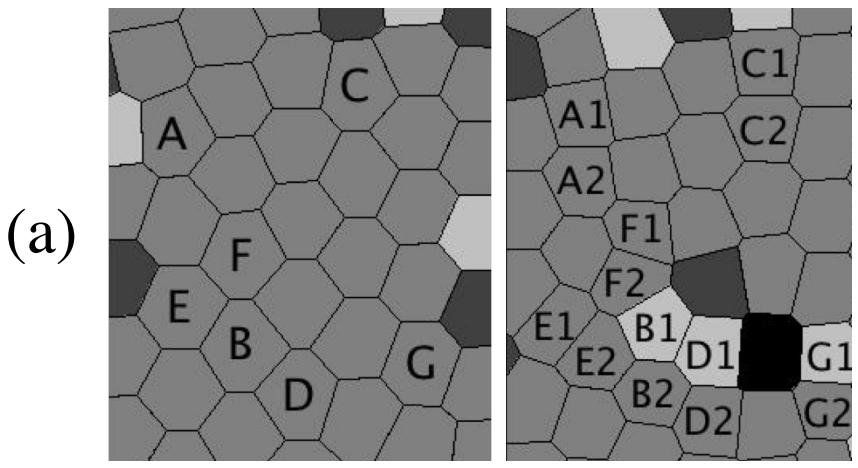

(b)
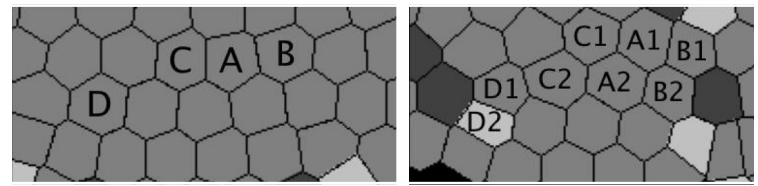
(a)
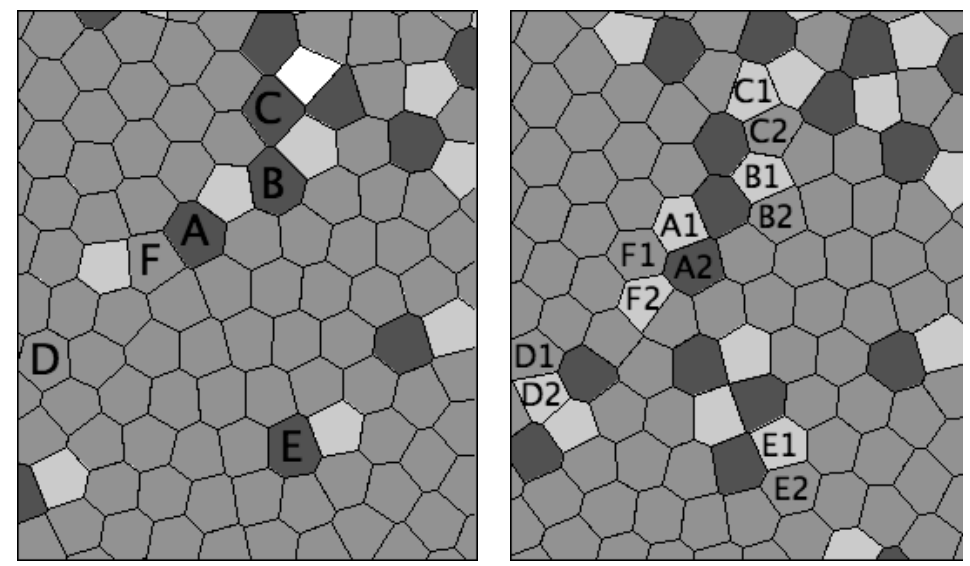

(b)
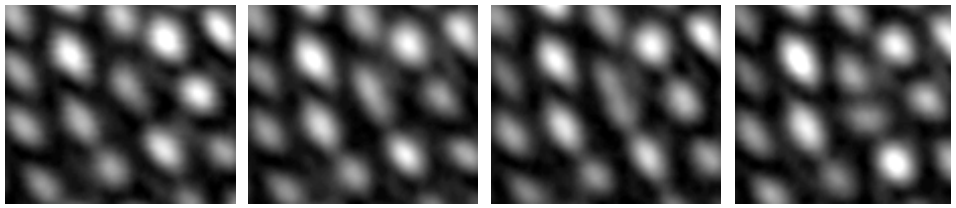


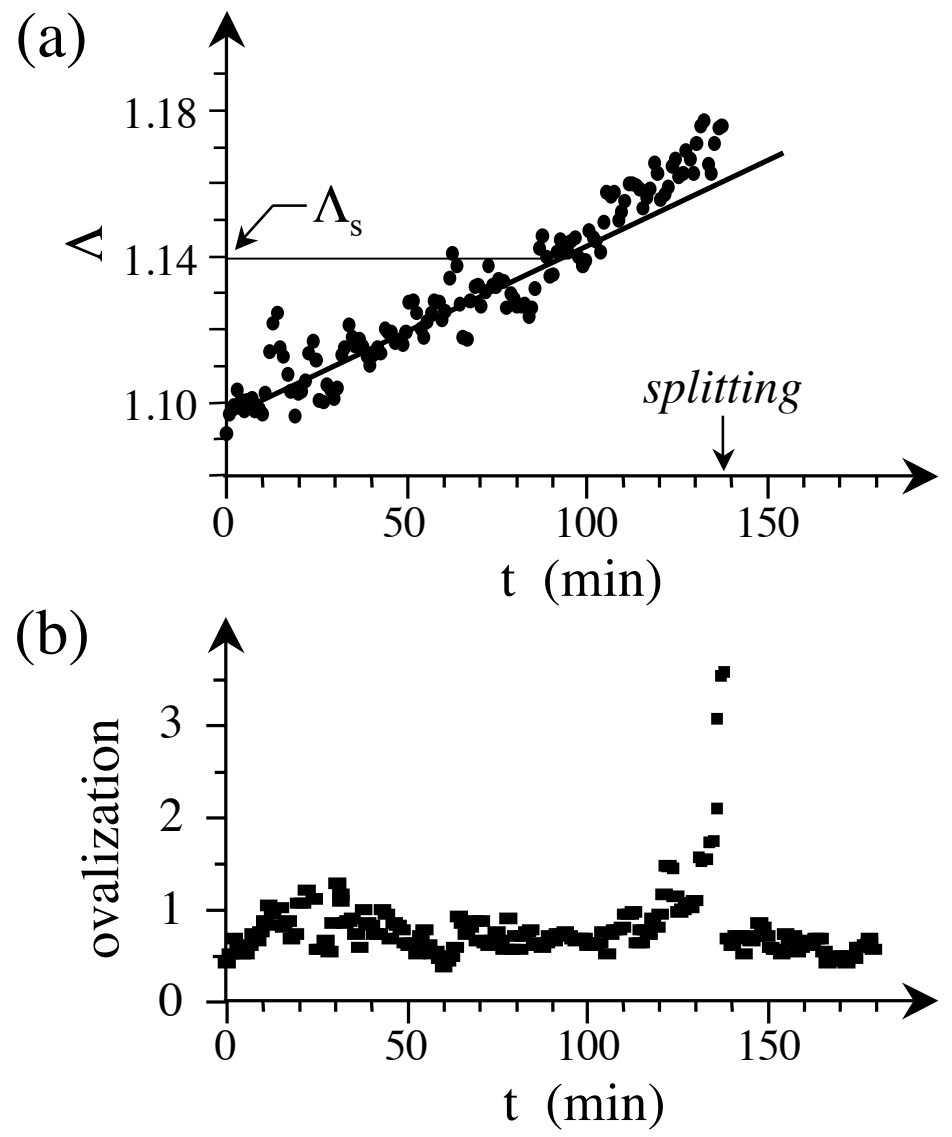




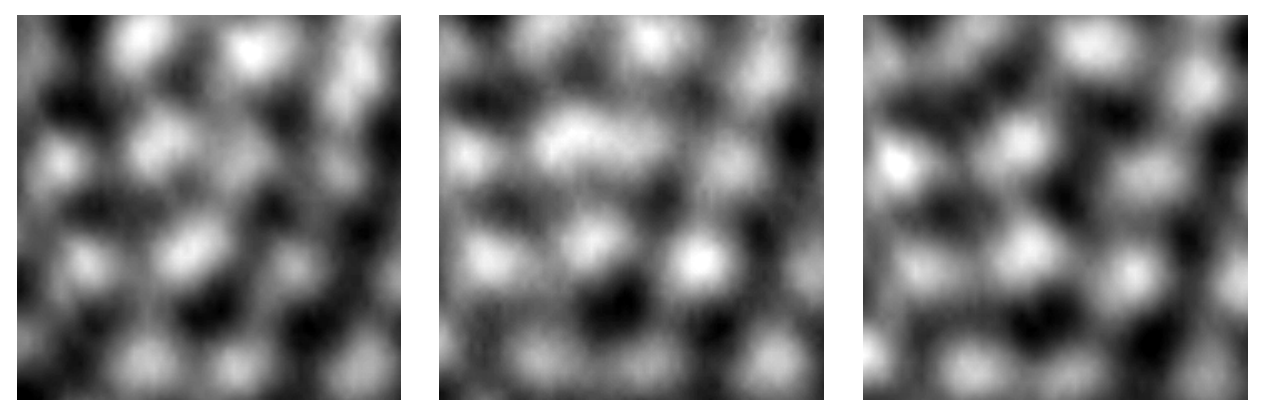




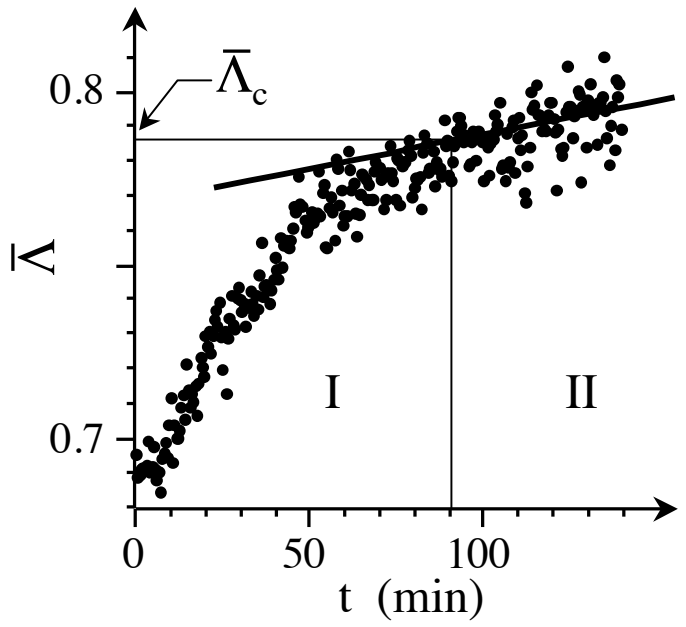




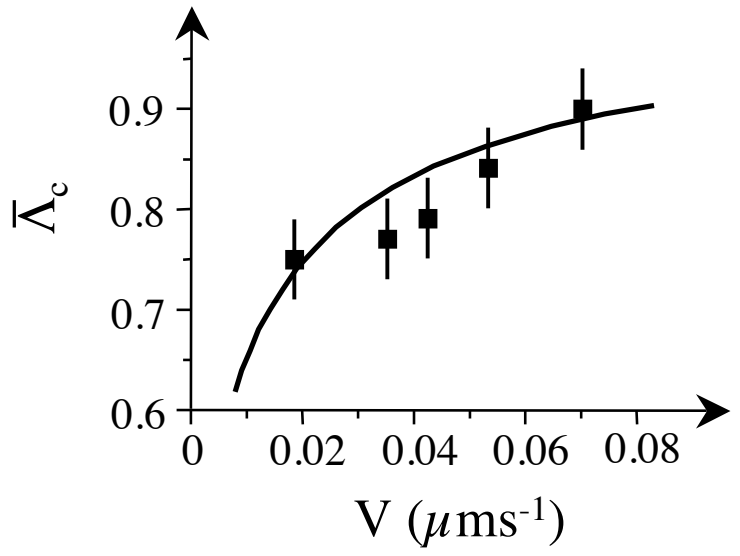



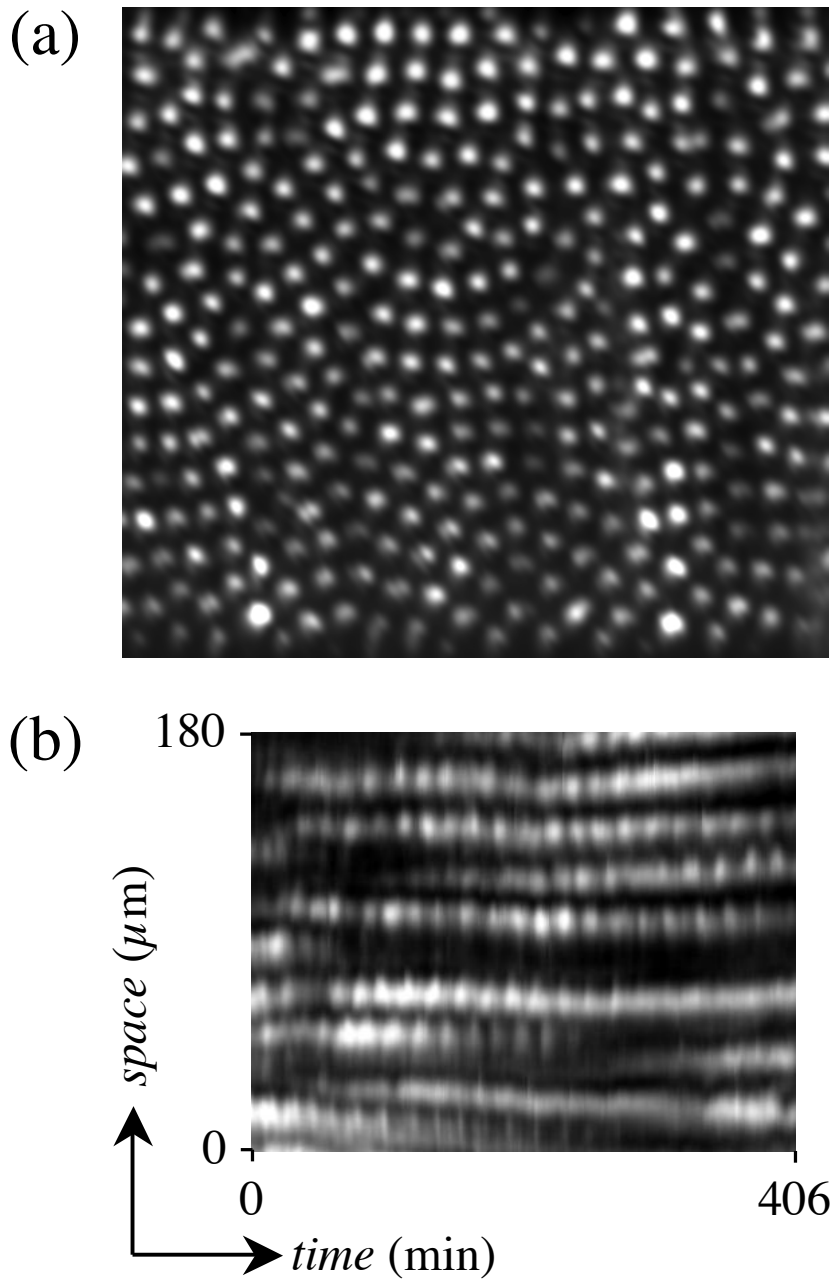


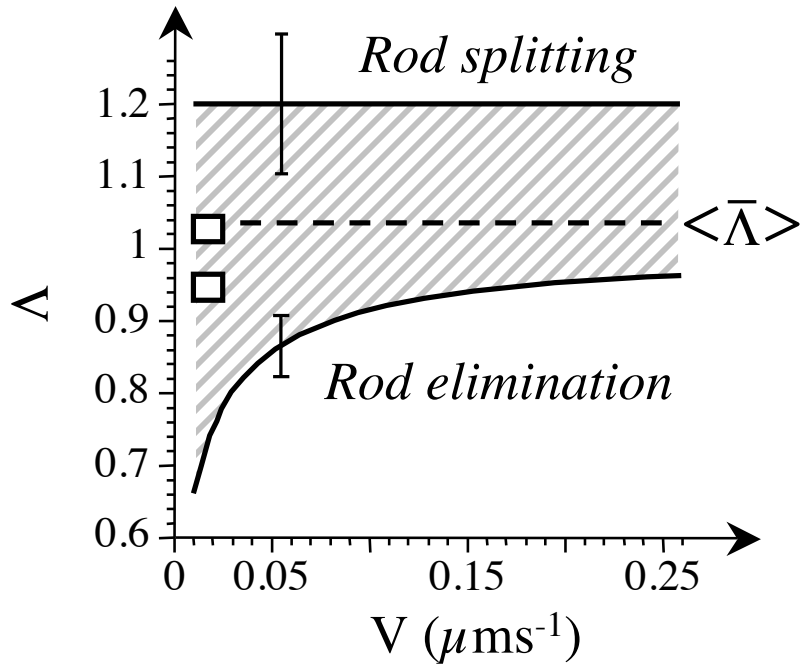

